\title{
Modeling and Design of Multi - Agent Logistics Robot Scheduling Simulation Platform
}

\author{
Ying Huang ${ }^{1, a, *}$, Jie Zhu ${ }^{1, b}$ \\ ${ }^{1}$ School of Information, Beijing Wuzi University, Beijing 101149, China. \\ a huangying0126@163.com, ${ }^{\mathrm{b}}$ zhujie@bwu.edu.cn
}

\begin{abstract}
This paper mainly analyzes scheduling business of the logistics distribution center, and according to the scheduling business workflow abstracts several kinds of agents, and clears their responsibilities. Based on the distributed multi-agent architecture, the logical architecture of the three-layer logistics robot scheduling simulation platform is designed and the middleware framework model is given.
\end{abstract}

Keywords: multi-agent system, logistics scheduling, simulation platform, middleware.

\section{Introduction}

With the rapid development of computer and communication technology, logistics has become the enterprise "third profit source". The logistics dispatching system can reflect the supply and demand of the materials and products in the process of production and operation of the enterprise and give the low cost and short scheduling period plan to guide the enterprise decision-making and management planning and arrange the production activities so as to reduce the system cost, and comprehensively improve the core competitiveness of enterprises [1].

At present, the international community has a variety of Agent-based simulation platform, such as Swarm of the United States Santa Fe Institute of the simulation platform; SourseForge Repast project; Ascape of University of Chicago Social and Economic Dynamics Research Center; StartLogo of Massachusetts University of Technology Media Labs and so on. Domestic research on the agent-based simulation platform, there are Li Hongliang developed Jclass, but also belong to the "laboratory-level products." However, due to the limitations of developers and the development of computer technology, many agent-based simulation platforms are inherently limited, and simulation of complex problems is difficult. How to improve the flexibility, reusability and scalability of the simulation platform becomes an important issue to research simulation platform.

\section{Multi - agent system overview}

Agent is a computer program that interacts with the environment and takes an autonomous act to accomplish a given goal. Agent has many characteristics, such as: autonomy, interactivity, reactivity, reasoning, adaptability and so on. Now the study of agent model can be divided into three types: cautious type, reactive type, mixed type. Multi-agent system refers to some autonomous agents through the collaborative work to complete some of the task of the computing system; it can be deepened the problem of deep decomposition into simple tasks, making the system easy to upgrade and control; and it can accept dynamic and uncertain knowledge, with a certain degree of intelligence, adaptability and robustness. It's very suitable for distributed architecture. These good properties make many observers think that Agent is the most important new example of software development after object-oriented, which has long-term significance for IT industry's competitiveness and survivability. 


\section{The function of each agent in the scheduling system}

After detailed investigation of the electricity supplier distribution center's order interaction issues, this article based on the principle of workflow analysis process of order processing, established the scheduling system framework, as shown in Figure 1.

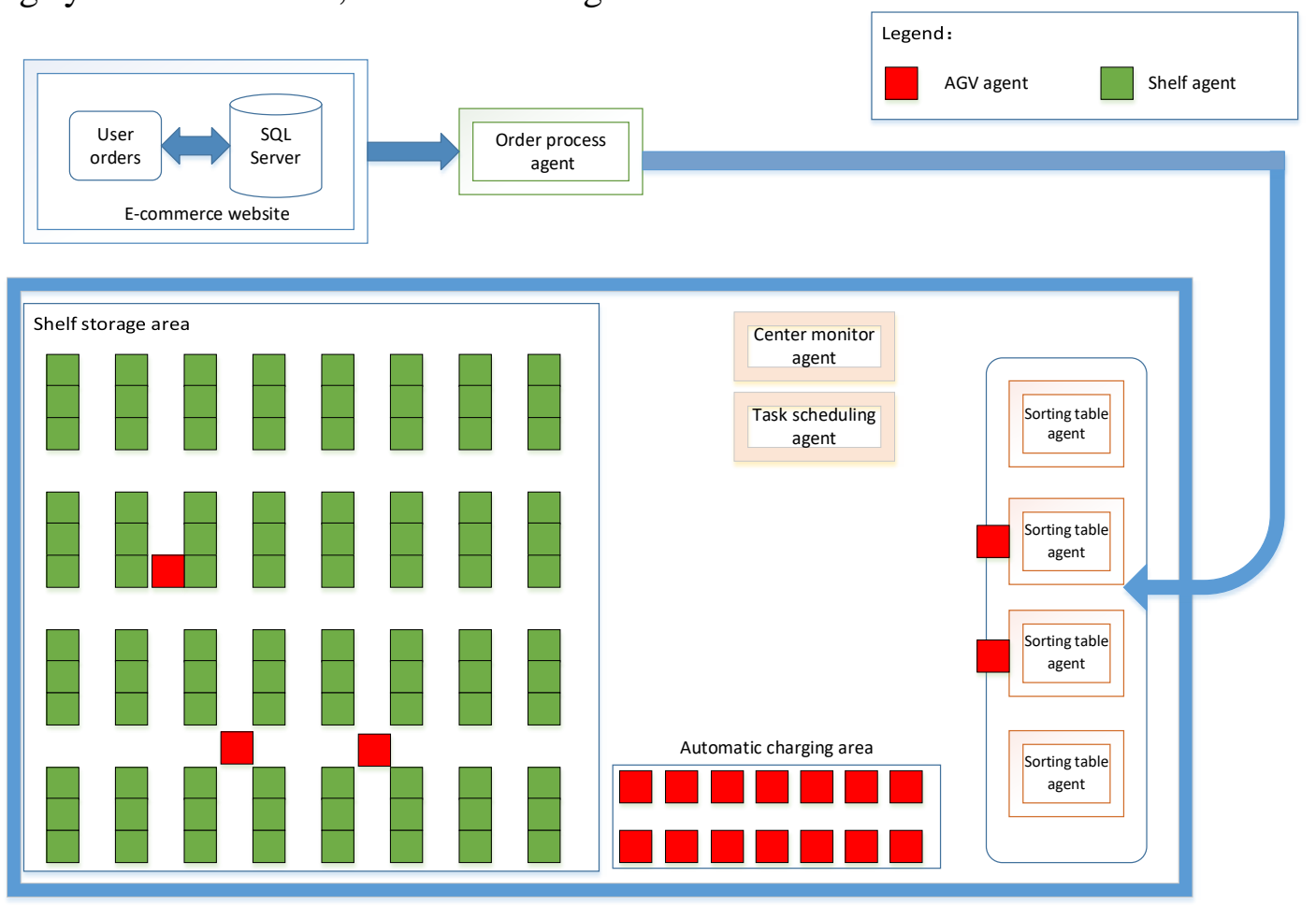

Fig. 1 Scheduling system framework

After analyzing the scheduling workflow, some elements that participate in the scheduling work are abstracted into various agents and give different responsibilities. In the framework, the user purchases the merchandise from the e-commerce website and orders, the orders will be stored in the database; the order process agent at every given time to remove the period of time has not been selected orders, according to established selection rules (such as order priority, product coincidence, etc.) to the preliminary analysis of the classification, then issued to the sorting table agent; after sorting table agent received a certain class orders or replenishment orders, dismantle this class order, and generate a task order, then assign the task to the task scheduling agent. The task scheduling agent distributes task to the corresponding AGV agent through the bidding method based on the contract network communication protocol, and the AGV agent completes the handling task.

The detailed functions of each Agent are described as follows:

(1)Road Agent (Node agent, Link agent).Dividing the road into two parts of Node and Link is more conducive to AGV agent and road agent interactive communication. In general, the intersection, the corners are divided into Node, and in addition to the other part of the straight line is divided into Link. Node and Link have the following basic properties, as shown in Table 1. 
Table 1 Node/Link attribute table

\begin{tabular}{|c|c|}
\hline \multicolumn{2}{|l|}{ NODE } \\
\hline ID & Node ID \\
\hline X coordinate & Position X \\
\hline Y coordinate & Position Y \\
\hline Enter the road section & Approach Links \\
\hline Exit the road section & Departure Links \\
\hline \multicolumn{2}{|l|}{ LINK } \\
\hline ID & Link ID \\
\hline Rode Name & Name \\
\hline Entrance Node & Entrance Node \\
\hline Exit Node & Exit Node \\
\hline Road section type & Type \\
\hline Road section length & Length \\
\hline
\end{tabular}

The interaction between AGV agent and Link agent is the most critical and frequent interaction process. When the AGV agent enters a link, it needs to register with the Link agent so that the Link agent knows the existence of the $\mathrm{AGV}$, and the Link agent will start monitoring the location information of the AGV and pass the corresponding information to the AGV agent. When the AGV agent leaves a link, it also sends a message to the Link agent to let the Link agent log off the registration of the AGV agent. When the AGV reaches, drives on and leaves the Node, it should be interactive cooperation with the Node agent. AGV agent needs to get the corresponding information from the Node, for their own path planning to provide decision support [2].

(2)AGV Agent. AGV agent is responsible for the successful bid to receive and complete their respective handling tasks. AGV agent includes the definition of physical and behavioral characteristics.

Physical properties: AGV's physical size, maximum speed, maximum acceleration and other dynamic characteristics.

Behavioral characteristics: AGV need according to certain rules to move, in the provisions of the road in accordance with a certain direction. AGV's driving behavior can be broken into the forward, acceleration, deceleration, stop, avoid and other basic behavior.

(3)Task Scheduling Agent. The task scheduling agent is responsible for receiving the handling task sent by the sorting table agent and matching with the AGV agent and generating the AGV transport path.

(4)Center Monitor Agent. The central monitor agent is the state management and information interaction core of the whole Multi-Agent system, and records the real-time status of each agent.

(5)Order Process Agent. The order process agent obtains the order or replenishment order at the pre-set time point. According to the pre-set principle, such as the order priority, the goods coincidence degree and so on to preliminarily analyze and classify the order, then send the order or replenishment order to the sorting table agent.

(6)Sorting Table Agent. The sorting table agent is responsible for receiving a certain order or replenishment order and disassembling to generate a handling task and then sending it to the task scheduling agent.

(7)Shelf Agent. The shelf agent is responsible for monitoring cargo dynamics, margin and other information at real-time, then according to the default alarm value issued out of stock warning and generate replenishment orders.

\section{Structure design of simulation platform}

\subsection{Logical Architecture Design}

The simulation platform from the distribution center of the actual work needs, and it is divided into three layers: order acquisition layer, scheduling layer and device layer, as shown in Figure 2. 


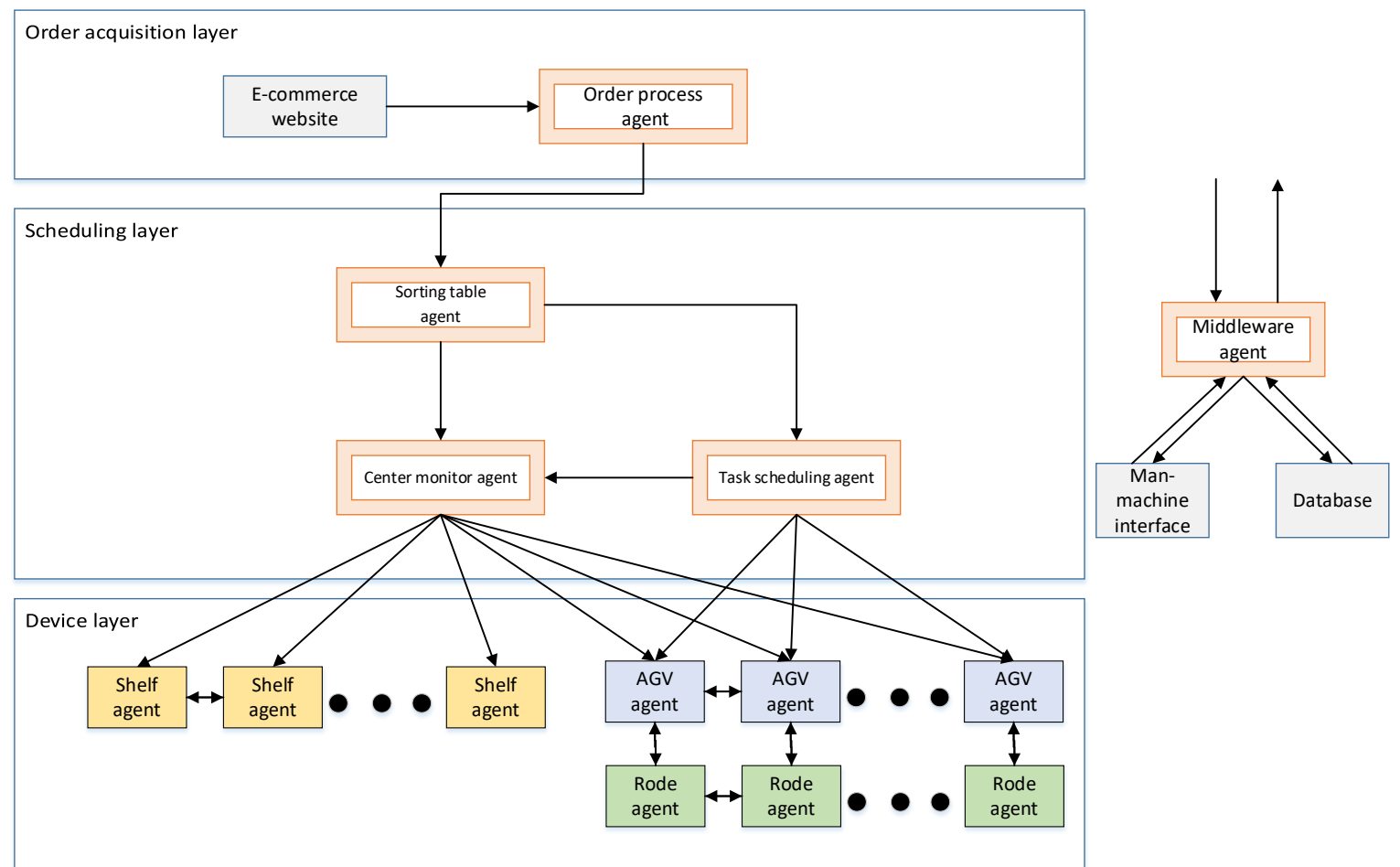

Fig. 2 Design of Logical Architecture for Dispatching Simulation Platform

All manuscripts must be in English, also the table and figure texts, otherwise we cannot publish your paper. Please keep a second copy of your manuscript in your office. When receiving the paper, we assume that the corresponding authors grant us the copyright to use the paper for the book or journal in question. Should authors use tables or figures from other Publications, they must ask the corresponding publishers to grant them the right to publish this material in their paper.

The main functions of each layer are as follows:

Order acquisition layer: The order acquisition layer is composed of the order processing agent, and the relevant order data of the e-commerce website outside the simulation platform is integrated. Through acquisition and integration of the external order, it delivery of the order data that the next level required to the next layer.

Scheduling layer: The scheduling layer mainly includes sorting table agent, task scheduling agent and central monitor agent. In this layer, the agent interacts with each other through the way of transmitting the message, and the decomposition of the order and AGV scheduling are realized.

Device layer: The device layer includes all controllable shelf agents, AGV agents, and road agents in the automated warehouse. These agents receive the data from the upper layer agent and combine the negotiation mechanism of itself to complete the scheduling task of the whole system.

In the process of the three-tier operation, the middleware agent into the various processes to complete the platform to achieve human-computer interaction and related to the dynamic display of data [3].

\subsection{Contract Network Consultation Process}

The task scheduling agent receives the handling task sent by the sorting table agent and needs to be assigned to each idle AGV agent to perform. They use the contract network agreement for consultation. There are two roles which are managers and contractors in the classic contract network agreement. In this simulation platform model, the task scheduling agent is the managers, and each AGV agent is the contractor, their specific consultation process is as follows:

Tender: After the sorting table agent sends the order data to the task scheduling agent, the task scheduling agent sends the AGV agent to complete the handling task through the communication channel in the form of issuing the tender, and requests them to complete the handling task. Bid: AGV agent receives the tender, and combined with its own real-time operation, and according to their own intent to consider, then makes respond to the received tender, and replies to the task scheduling 
agent whether participate in the implementation of the task. Finally, the entire bidding process is completed. Winning bid: The task scheduling agent completes the operation of the related optimization algorithm according to its own objective function, and selects the highest efficiency one from the received tender, then assigns the task to the winning AGV agent, and sends the other AGV agent to failure information. Implementation: AGV agent which received the bid tag performs the task that the task scheduling agent sent to. After a control cycle, regardless of whether the task is completed successfully, the implementation of the results need to be passed to the task scheduling agent, as the task scheduling agent next round of coordination of work reference data.

\subsection{Simulation Agent Model Based on BDI}

BDI thought is widely used in Agent's logical layer modeling, which is an effective method for agent to realize logic.

B (Belief): it represents the belief, and mainly descripts knowledge system that agent has, and it is the basis for decision-making; D (Desire): it's a description of the purpose of the agent, which represents the desired outcome of the agent behavior; I (Intention): it represents the current intention of the agent, that agent expected to perform the act, and it is served by Desire. In the BDI system, above three are linked which is BDI reasoning, the so-called BDI reasoning is: Agent knowledge (Belief) as the basis, desire as the guide, reasoning Agent behavior (Intention).

The standardization of BDI thought is as follows:

$$
\begin{aligned}
& \text { B: }=\operatorname{rec}(B, e) \\
& \text { D: }=\bmod (B, D) \\
& \text { I: }=\operatorname{sele}(D)
\end{aligned}
$$

The first formula indicates that Agent obtains information from the outside world, and then integrates with the original knowledge (Belief) to obtain new knowledge (Belief). The second formula indicates that the Agent obtains a new desire (Desire) according to the change of the environment (which is manifested in the change of knowledge) and the previous desire (Desire).The last formula indicates that Agent acts to select action according to desire (Intention).The above operation is the process of continuous circulation until the Agent achieves the goal or satisfies the ending condition.

The agent that implements BDI thought in abstract hierarchical way is the typical BDI agent. On this basis, a general framework for scheduling simulation agent modeling is proposed: First, the agent is abstracted into the social layer, the logical layer and the task layer. Where the social layer is responsible for communication and the logical layer is implemented using BDI ideas. The task layer consists of a number of meta-modules that perform specific functions that can be combined to achieve specific complex behavior. Following this principle, when modeling the simulation platform we just focus on the definitions of the B, D, I set, establishment of BDI logic and implementation of meta-functional module. The general BDI agent model is shown in Figure 3. 


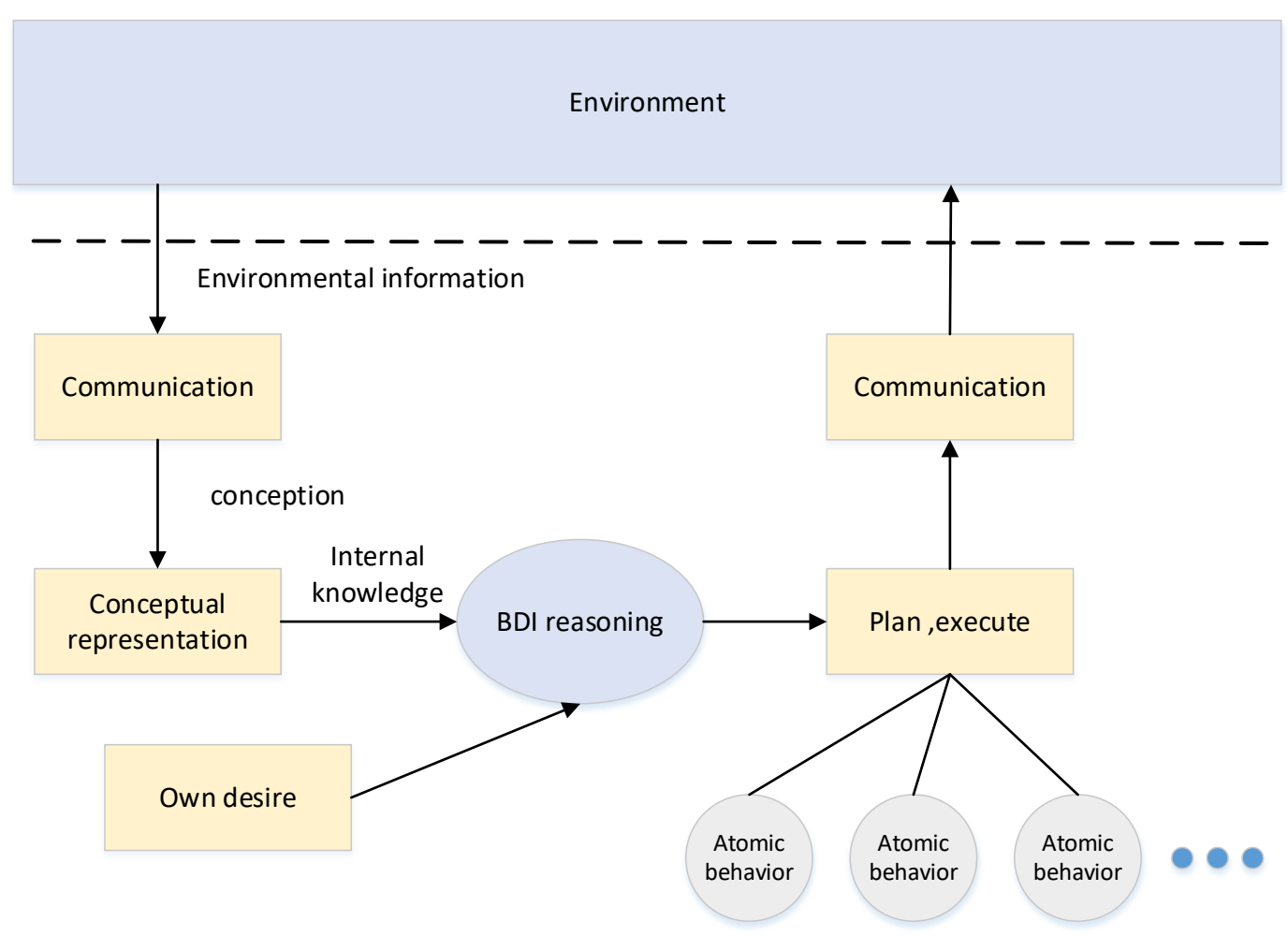

Fig. 3 General BDI agent model

The figure above shows the behavior model of the BDI agent in a cycle. It is divided into the following steps: Agent perceives the environment and obtains environmental information by means of communication. Agent deals with environmental information, and expresses it as a concept, and adjusts its desire when the environment changes. Agent generates a plan based on its own desires and BDI reasoning rules. Agent executes the generated plan. When the plan is completed, it will report to the environment the new state.

The core of this generic model is the part associated with BDI, whose mathematical model is as follows: Define the knowledge set of the agent $K=\left\{k_{1}, k_{2}, k_{3}, \cdots \cdots, k_{n}\right\}$, for $\forall k_{i} \in K$, $k_{i}$ represents some knowledge required by the agent. As for the AGV agent, the knowledge it needed includes other AGV information, road information, handling task information and so on. Define the desire set of the agent $D=\left\{d_{1}, d_{2}, d_{3}, \cdots \cdots, d_{n}\right\}$, for $\forall d_{i} \in D, d_{i}$ represents the agent's desire. As for the AGV agent, one of its desire may is, from the current location of the warehouse map to reach the shelf position needed to be carried. Define the atomic behavior of the agent $B=\left\{b_{1}, b_{2}, b_{3}, \cdots \cdots, b_{n}\right\}$, for $\forall \mathrm{b}_{\mathrm{i}} \in \mathrm{B}, \mathrm{b}_{\mathrm{i}}$ represents the atomic behavior of the agent. As for the AGV agent, its atomic behavior can include acceleration, deceleration, stop, turn left, turn right and so on. Based on the combination of these atomic acts, it can get a complex higher level behavior. Define the Intention set of the agent $\mathrm{I}=$ $\left\{\mathrm{i}_{1}, \mathrm{i}_{2}, \mathrm{i}_{3}, \cdots \cdots, \mathrm{i}_{\mathrm{n}}\right\}$, for $\forall \mathrm{i}_{\mathrm{k}} \in \mathrm{i}, \mathrm{i}_{\mathrm{k}}$ represents the current intention of the agent. As for the AGV agent, it is currently possible including the avoidance, changing the path and other intentions. Define the combination of $i_{k}$ and $B, i_{k}=b_{k 1} \rightarrow b_{k 2} \rightarrow b_{k 3} \cdots \cdots b_{k j}, i_{k} \in I, b_{k n} \in B(1 \leq n \leq j), i_{k}$ is obtained by combining elements in $\mathrm{B}$ in a certain order, there may also be some parameters. Define the BDI reasoning function $R\left(K, d_{i}\right)=i_{j}, d_{i} \in D, i_{j} \in I$. This formula shows that the agent-based desire and knowledge, agent get next step through BDI reasoning. K only represents that BDI reasoning needs to be based on the knowledge set, when actually implement it, you can first deal with the knowledge set, and get some conclusions or logical signs, which as parameters send to the function $\mathrm{R}$, and then do not need to analyze the entire knowledge set at the R.

\subsection{The Design of Middleware}

In this paper, the design idea of software agent is used in the design of middleware, which can make the middleware have the characteristics of intelligence and cooperation [4,5]. The middleware agent model given is shown in Figure 4. 


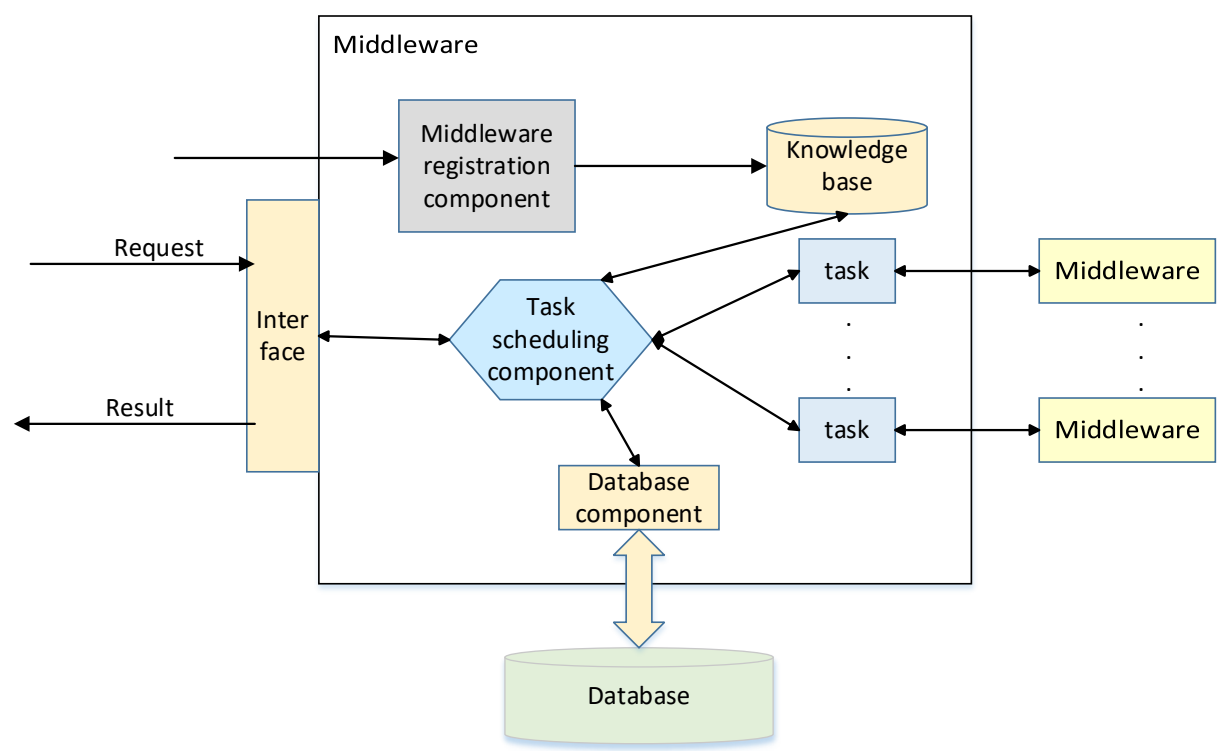

Fig. 4 Structure diagram of Middleware agent

Mainly consists of the following five parts: (1) middleware interface: to provide users with the task request, and return the results; (2) middleware registration components: it equals to external world sensor components of the agent, and accepts other middleware information. Register or update in the knowledge base to lay the foundation for interaction with other middleware, building middleware collaborative environment; (3) database components: the operation that middleware for database all packaged in this component, including database connectivity, query, implementation and other statements; (4) task scheduling components: middleware task scheduling component is the core of the middleware. After middleware received an external request, according to the contents of the knowledge base to generate the task module to complete the task, and combining with the results returned to the external request; (5) task components: task scheduling components generate the entity to complete the task, it can complete the task within the middleware, also can interact with other middleware to complete the task.

\section{Conclusion}

This paper analyzes the current logistics scheduling business process, and establishes the middleware model based on the distributed multi-agent system and the agent model in the dispatching system. Although the establishment of these models is for the logistics scheduling business, but at the same time for other business systems simulation research of the logistics business also has a strong reference. Through the establishment of middleware and agent model, and then build a logistics robot scheduling simulation platform based on distributed multi-agent system, it can use to perform simulation experiments for the existing scheduling business applications.

\section{Acknowledgements}

This paper is supported by the Funding Project for Technology Key Project of Municipal Education Commission of Beijing (ID:TSJHG201310037036); Funding Project for Beijing key laboratory of intelligent logistics system; Funding Project of Construction of Innovative Teams and Teacher Career Development for Universities and Colleges Under Beijing Municipality (ID:IDHT20130517), and Beijing Municipal Science and Technology Project (ID:Z131100005413004);Funding Project for Beijing philosophy and social science research base specially commissioned project planning (ID:13JDJGD013); Funding Project for Beijing Intelligent Logistics System Collaborative Innovation Center. 


\section{References}

[1] Zhai Guimin. Multi-agent logistics scheduling system modeling and design [J]. Modern computer (Professional Edition), 2011, (26): 67-70.

[2] Xu Zhen. Vehicle modeling of multi-agent micro-traffic simulation under HLA simulation framework [J]. Shanghai Jiao Tong University, 2008.

[3] Wang Kangyuan. Multi-agent based wind power generation active power control simulation platform [D]. North China Electric Power University, 2016.

[4] LU Xiong-bin, GUO Chao-zhen. Study and application of cloud service middleware based on multi-agent [J]. Microcomputer and Application, 2013, (20): 87-90.

[5] Feng Feng, Xu Qi. Research on e-commerce system based on mobile agent middleware [J]. Manufacturing Automation, 2011, (17): 24-27 + 79 . 